\title{
Review
}

Neuropsychobiology

\section{Continuation and Maintenance Electroconvulsive Therapy for Mood Disorders: Review of the Literature}

\author{
Georgios Petrides $^{a} \quad$ Kristen G. Tobias $^{b} \quad$ Charles H. Kellner ${ }^{b}$ \\ Matthew V. Rudorfer ${ }^{\mathrm{c}}$ \\ ${ }^{a}$ The Zucker Hillside Hospital, North Shore-LIJ Health System, Glen Oaks, N.Y., b The Mount Sinai School of Medicine, \\ New York, N.Y., and 'National Institute of Mental Health, Bethesda, Md., USA
}

\section{Key Words}

Electroconvulsive therapy - Continuation electroconvulsive therapy · Maintenance electroconvulsive therapy $\cdot$ Mood disorders $\cdot$ Review

\begin{abstract}
Background: Electroconvulsive therapy (ECT) is a highly effective treatment for mood disorders. Continuation ECT (C$E C T$ ) and maintenance ECT (M-ECT) are required for many patients suffering from severe and recurrent forms of mood disorders. This is a review of the literature regarding $C$ - and M-ECT. Methods: We conducted a computerized search using the words continuation ECT, maintenance ECT, depression, mania, bipolar disorder and mood disorders. We report on all articles published in the English language from 1998 to 2009. Results: We identified 32 reports. There were 24 case reports and retrospective reviews on 284 patients. Two of these reports included comparison groups, and 1 had a prospective follow-up in a subset of subjects. There were 6 prospective naturalistic studies and 2 randomized controlled
\end{abstract}

\section{KARGER}

Fax +4161306 1234

E-Mail karger@karger.ch

www.karger.com
(C) 2011 S. Karger AG, Basel

Accessible online at: www.karger.com/nps trials. Conclusions: C-ECT and M-ECT are valuable treatment modalities to prevent relapse and recurrence of mood disorders in patients who have responded to an index course of ECT. C-ECT and M-ECT are underused and insufficiently studied despite positive clinical experience of more than 70 years. Studies which are currently under way should allow more definitive recommendations regarding the choice, frequency and duration of C-ECT and M-ECT following acute ECT.

Copyright $\odot 2011$ S. Karger AG, Basel

\section{Introduction}

More than 70 years since its introduction, electroconvulsive therapy (ECT) remains the most effective somatic treatment in psychiatry, with unsurpassed efficacy and remarkable safety. ECT is effective for various conditions and is a viable treatment option when pharmacotherapy and psychotherapy have failed, when affective, psychotic or catatonic symptoms are present, and when rapid relief 
of symptoms is required because of suicide risk or deterioration of medical conditions [1-6]. ECT is most commonly used for the treatment of severe depression, but is also effective for the treatment of manic or mixed episodes [7-9].

Many patients with mood disorders, for which treatment with ECT is indicated, suffer from conditions that are chronic and recurrent in nature. Continuation therapy after remission of an acute episode of a mood disorder is considered to be the standard of practice in modern psychiatry [10]. Following successful treatment with acute ECT, continuation therapy is of particular importance, as these patients frequently have the most severe, recurrent and treatment-resistant illness. A study by Sackeim et al. [11] demonstrated that the relapse rate of patients with unipolar depression who remit after an acute course of ECT is extremely high if there is no active treatment after the last ECT. Patients who received placebo relapsed at a rate of $84 \%$ within 6 months after acute ECT. Patients who received monotherapy with the tricyclic antidepressant nortriptyline relapsed at a rate of $60 \%$, and those receiving combination therapy consisting of nortriptyline and lithium relapsed at a rate of $39 \%$. These results underscore the need for aggressive preventative approaches to sustain the clinical benefits of acute treatment.

When ECT is used for the treatment of an acute episode, it is reasonable to consider continuation ECT (CECT) or maintenance (M-ECT) to prevent relapse of the current episode or recurrence of a new episode. The term 'continuation ECT' (C-ECT) and 'maintenance ECT' (MECT) are frequently used interchangeably and indiscriminately across the mood disorder treatment continuum. For the purpose of this report, we will use the following definitions: an index/acute course is the initial series of treatments given for the purpose of relieving acute symptoms of the illness. C-ECT is a course that begins after the index course, lasts up to 6 months, and is designed to prevent relapse of the episode (return of the symptoms to full syndromal criteria before the end of the natural duration of the illness). $M$-ECT is a course that begins after the end of C-ECT and is intended to prevent recurrence of an episode (a new episode).

The practice of C-ECT and M-ECT has been documented almost since the introduction of ECT by Cerletti and Bini in 1938 [12, 13]. However, it has not always been widely implemented in clinical practice during the psychotropic medication era of modern psychiatry. The development of antipsychotic and antidepressant medications in the 1950s created the belief that ECT had been superseded, and the practice of ECT declined dramatically. This period, which lasted until the early 1970s, was characterized by the polemics of the antipsychiatry movement which attacked psychiatry in general and ECT in particular [14]. ECT, despite its decline in numbers, was never abandoned, as many patients did not respond to medications and psychotherapy. However, the negative climate prompted many psychiatrists to recommend as few treatments as possible, often limiting the number of ECT treatments to the absolute minimum to achieve improvement of acute symptoms, thus automatically excluding C-ECT and M-ECT from their armamentarium. In 1974, the American Psychiatric Association (APA) appointed a Task Force to study and report on '[ECT] legislative issues, consent, indications for its use and possible increasing use ...' [15]. The Task Force reported that there was a role for ECT in the treatment of depression, intractable mania and treatment-resistant schizophrenia [15]. There was no particular mention of C-ECT in that report, yet interest in the practice of ECT was restimulated.

A survey of practice by Kramer [16] conducted in 1986 revealed that many psychiatrists were treating their most treatment-resistant patients with C-ECT. C-ECT was first mentioned in the 1990 APA Task Force report [17]. The third and latest APA Task Force Report (2001) [3] established the indications for C-ECT and M-ECT for patients who responded to an acute ECT course when one of the following has occurred:

(1) pharmacotherapy alone has not been effective in treating index episodes or in preventing relapse or recurrence;

(2) pharmacotherapy cannot be safely administered, or

(3) the patient prefers treatment with ECT and the patient or surrogate consentor agrees to the patient's receiving $\mathrm{C}$-ECT. The patient must be capable, with the assistance of others, of complying with the treatment plan. The Association for Convulsive Therapy (ACT), a professional society of ECT clinicians and researchers, created a task force that recommended guidelines for the practice of ambulatory ECT [18]. As most C-ECT and MECT is performed on an outpatient basis, the majority of these guidelines are applicable and should facilitate this practice. Nevertheless, as we pointed out in an earlier review [19], there is little early systematic research on CECT and M-ECT. Many case reports and several studies appeared in the literature until 1965, attesting to the utility of ECT courses given beyond the point of acute treatment [20]. Inherent weaknesses of these studies, as in most of the psychiatric literature of that era, include the use of heterogeneous populations and weak methodology. 
One would have expected a much greater number of research publications on C-ECT and M-ECT over the course of more than 7 decades, especially considering the almost unanimously positive attestations of the reports. This lack of literature between 1965 and 1985 reflects, besides the decline in the use of ECT, the fact that C-ECT is underused and that research resources directed to the study of ECT are limited. One cannot underestimate the effects of the negative public environment regarding ECT on scientific thinking and directions of scientific research. The stigma against ECT is perpetrated even by professionals, as evidenced by the National Institute for Clinical Excellence (NICE) report in the UK, which disputed the utility of C-ECT and M-ECT in its 'Guidance on the Use of Electroconvulsive Therapy' [21], despite positive recommendations from the APA Task Force on ECT and protest from British psychiatrists [22-24]. We responded to this report with a paper in the Journal of ECT [25] which documents the logical inconsistencies in the flawed British report.

With the renewal of interest in ECT, more reports about C-ECT were published during the last two decades. These studies reconfirm the benefits of ECT given beyond the index course. Unfortunately, most of these reports are retrospective and describe only a small number of patients. Prolonging remission after successful acute treatment with ECT remains an important clinical challenge. If C-ECT is implemented, common clinical practice includes a taper and then treatments spaced out at gradually increasing intervals. However, there is a dearth of guidance about the optimal frequency of C-ECT, concurrent pharmacotherapy and the overall tolerability of C-ECT.

At the time of our earlier review in 1997, C-ECT and M-ECT were not often considered as options. However, newer research and experience have established C-ECT and M-ECT as important tools for relapse prevention in patients who have responded to ECT for the treatment of an acute episode of a mood disorder. The aim of this report is to review the literature on C-ECT and M-ECT after 1997 and to delineate evidence-based guidelines for its safe and effective practice.

\section{Methods}

A PubMed search was conducted using the words 'continuation ECT', 'maintenance ECT', 'depression', 'mania', 'bipolar disorder' and 'mood disorders'. We report on all articles published in the English language from 1998 to 2009.

\section{Results}

We identified 32 reports. There were 24 case reports and retrospective reviews on 284 patients. Two of these reports included comparison groups, and 1 had a prospective follow-up component in a subset of subjects. Table 1 summarizes the published retrospective studies and case reports on C-ECT and M-ECT. There were 6 prospective naturalistic studies and 2 randomized controlled trials (RCTs). Table 2 summarizes the published literature on prospective studies. Of note, there are several reports that examine the use of C-ECT and M-ECT in various other disorders, such as schizophrenia and schizoaffective disorder, Parkinson's disease and autism, that are beyond the scope of this review and are not included here [26-34].

Most published studies are positive with regards to the efficacy and safety of C-ECT and M-ECT in mood disorders. As in our earlier review, the majority of these studies are uncontrolled prospective or retrospective studies, or case reports. However, a major addition to the literature is the National Institute of Mental Health (NIMH) funded multicenter RCT conducted by the Consortium for Research on ECT (CORE) [65]. This is the first large RTC to compare the relative efficacy of C-ECT to a combination pharmacotherapy regimen. In this well-designed study, 531 patients with unipolar major depressive disorder (with and without psychotic features) were enrolled into the acute ECT phase. ECT was performed with bifrontotemporal electrode placement at 1.5 times the determined seizure threshold. Of those patients 341 met remission criteria after an average of 6.8 ECT. After an interim week without additional treatment, 201 patients were enrolled into the continuation phase of the study and were randomized to receive 6 months of C-ECT or continuation pharmacotherapy (C-PHARM). C-ECT was given at a predetermined fixed schedule with 4 weekly treatments the first month, followed by 4 biweekly treatments for 2 months and 2 monthly treatments for a total of 10 ECT in 6 months. Patients in this arm received no psychotropic medications except for lorazepam or diphenhydramine on an as-needed basis. C-PHARM consisted of the combination of lithium and nortriptyline and patients were evaluated at the same interval as in the C-ECT arm. The relapse rates at 6 months did not differ statistically between the two arms $-37.1 \%$ for C-ECT and $31.6 \%$ for C-PHARM - and were comparable to those reported in the similarly designed study by Sackeim et al. [11] for the combination of lithium and nortriptyline (39\%), and far better than those reported for nortriptyline

Neuropsychobiology 2011;64:129-140 
Table 1. Retrospective studies and case reports on C-ECT effectiveness and tolerability (1998-2009)

\begin{tabular}{|c|c|c|c|c|c|c|}
\hline Author & Design & $\begin{array}{l}\text { Diagnostic } \\
\text { criteria }\end{array}$ & Subjects, $\mathrm{n}$ & $\begin{array}{l}\text { C-ECT type/ } \\
\text { duration/interval }\end{array}$ & Medication & Outcome/comments \\
\hline $\begin{array}{l}\text { Bonds } \\
\text { et al. [35] } \\
(1998)\end{array}$ & case report & $\mathrm{BD}$ & 1 & $\begin{array}{l}\text { BL/10 months/variable, } \\
\text { then monthly }\end{array}$ & $\begin{array}{l}\text { haloperidol for } \\
6 \text { of } 10 \text { months, } \\
\text { then no psychotro- } \\
\text { pic medication }\end{array}$ & $\begin{array}{l}\text { Psychotic depression, remained in remission. 'No adverse } \\
\text { medical complications and minimal cognitive side effects'. } \\
\text { Outpatient C-ECT cost-effective relative to 1-year compari- } \\
\text { son period of acute courses of ECT and hospitalizations. }\end{array}$ \\
\hline
\end{tabular}

Gupta case report BD $\quad$ BL/unspecified/unspecified lithium ( 0.77 mEq/l 'Mood and affect were much improved'. 'No delirium or oth-

at 2nd C-ECT) and er complications noted'. No self-report decline in memory as

$(1998)$

\begin{tabular}{lllll}
\hline $\begin{array}{l}\text { Kramer } \\
{[37]}\end{array}$ & case report & BD & 1 & $\begin{array}{l}\text { BL/3 years/individualized } \\
\text { to target at-risk seasonal } \\
\text { biological pattern }\end{array}$
\end{tabular} $\begin{aligned} & \text { lithium and } \\
& \text { clonazepam PRN }\end{aligned}$
measured by the SSMQ.

Kramer retrospective MDD, BD, $53 \quad$ 'most patients ... BL'/ unspecified

[38] review of a depression $+\quad 6$ months -4 years/variable

(1999b) university Axis II

ECT service diagnosis,

depression +

$\mathrm{PD}$

C-ECT effective in maintaining euthymia for long periods of time. Reduced hospitalizations relative to 4-year comparison period with pharmacotherapy alone. 'Memory loss was minimal.' imal.

18 of 24 patients with MDD experienced sustained improvement, 3 patients w/comorbid anxiety disorder had 'only partial response' even after more frequent C-ECT, 3 of 9 BD patients 'remained much improved', 7 of 9 had 'partial improvement', 1 of 10 patients with Axis II 'remained much improved', 6 of 10 patients with Axis II had a 'partial response,' PD patients had decrease in motor symptoms, '... no medical problems, other than headache and transient memory complaints'.

\begin{tabular}{|c|c|c|c|c|c|c|}
\hline $\begin{array}{l}\text { Gagne } \\
\text { et al. [39] } \\
(2000)\end{array}$ & $\begin{array}{l}\text { retrospective } \\
\text { review vs. } \\
\text { matched } \\
\text { pharmaco- } \\
\text { therapy } \\
\text { control group }\end{array}$ & MDD, BD & $\begin{array}{l}\text { C-ECT }+ \\
\text { C-PHARM } \\
=29 \\
\text { C-PHARM } \\
=29\end{array}$ & $\begin{array}{l}\text { unilateral }=8 ; \mathrm{BL}=19 \\
\text { crossover }=2 / \text { unspeci- } \\
\text { fied/' } \ldots \text { generally } \ldots \text { weekly } \\
\text { for the first month, every } 2 \\
\text { weeks for the following } \\
\text { month, and then monthly'. }\end{array}$ & $\begin{array}{l}\text { various } \\
\text { medications }\end{array}$ & $\begin{array}{l}\text { Following acute ECT, C-ECT + medication superior to med. } \\
\text { alone in preventing relapse ('... cumulative probability of sur- } \\
\text { viving without relapse ... } 93 \% \text { compared to } 52 \% \text { at year } 2 ; 73 \% \\
\text { compared to } 18 \% \text { at year } 5 \text {. Mean survival times were } 6.9 \\
\text { years for the continuation ECT patients and } 2.7 \text { years for the } \\
\text { antidepressant-alone patients.') }\end{array}$ \\
\hline $\begin{array}{l}\text { Chan- } \\
\text { pattana } \\
{[40](2000)}\end{array}$ & case report & $\begin{array}{l}\mathrm{BD} \text {, manic } \\
\text { episode }\end{array}$ & 1 & $\mathrm{BL} / 18$ months/variable & clozapine & $\begin{array}{l}\text { C-ECT + clozapine 'resulted in remission over an } 18 \text {-month } \\
\text { period.' The patient returned to his normal life and contin- } \\
\text { ued his academic studies.' }\end{array}$ \\
\hline $\begin{array}{l}\text { Stewart }[41] \\
(2000)\end{array}$ & case report & MDD & 1 & $\begin{array}{l}\text { BL/8 months/'every } 6 \\
\text { weeks' }\end{array}$ & $\begin{array}{l}\text { lithium } \\
\text { (level around } \\
0.7 \mathrm{mEq} / \mathrm{l} \text { ) }\end{array}$ & $\begin{array}{l}\text { Age } 78 \text {. 'Depressive symptoms remain in complete remission } \\
\text { after } 8 \text { months.' }\end{array}$ \\
\hline $\begin{array}{l}\text { Fox }[42] \\
(2001)\end{array}$ & case report & MDD & 3 & $\begin{array}{l}2 \text { BL } 1 \text { unknown/variable, } \\
>18 \text { months/variable }\end{array}$ & $\begin{array}{l}\text { various } \\
\text { medications }\end{array}$ & $\begin{array}{l}\text { Lifetime frequency, severity, and duration of relapse dimin- } \\
\text { ished by C-ECT, 'no medical complications', 'subjective } \\
\text { complaints of memory impairment were common'. At } 5-8 \\
\text { weeks after last C-ECT, } 2 \text { patients scored } 29 / 30 \text { on MMSE. }\end{array}$ \\
\hline $\begin{array}{l}\text { Kho }[43] \\
(2002)\end{array}$ & case report & RCBD & 1 & $\begin{array}{l}\mathrm{BL} \text { and unilateral/ }>1 \text { year/ } \\
4 \text { weeks }\end{array}$ & $\begin{array}{l}\text { lithium added } \\
(0.8 \mathrm{mmol} / \mathrm{l})\end{array}$ & $\begin{array}{l}\text { Age } 79, \text { C-ECT improved mood stability 'without ill effect to } \\
\text { her (frail) physical condition', lithium alone not sufficient for } \\
\text { mood stability. After } 51 \text { ECTs, MMSE 13/30. }\end{array}$ \\
\hline $\begin{array}{l}\text { Russell } \\
\text { et al. [44] } \\
(2003)\end{array}$ & $\begin{array}{l}\text { retrospective } \\
\text { review }\end{array}$ & $\mathrm{MDD}, \mathrm{BD}$ & 38 & $\begin{array}{l}\text { BT }(85 \%) ; \text { RUL }(12 \%) \\
\text { BF }(3 \%) / 27.8 \text { months } \\
\text { (mean); } 12-61 \text { months } \\
\text { (range)/variable }\end{array}$ & $\begin{array}{l}\text { various } \\
\text { medications }\end{array}$ & $\begin{array}{l}\text { '... sustained initial post-index ECT depression ratings and } \\
\text { even a slight drop over time, improved functional status, and } \\
\text { no cognitive deterioration' (as measured by the MMSE, } \\
\mathrm{HRSD}_{24} \text {, GAF). Reduced hospital days relative to compari- } \\
\text { son period of } 1 \text { year w/out C-ECT. }\end{array}$ \\
\hline $\begin{array}{l}\text { Vaidya } \\
\text { et al. [45] } \\
(2003)\end{array}$ & $\begin{array}{l}\text { retrospective } \\
\text { review }\end{array}$ & $\mathrm{BD}$ & 12 & $\begin{array}{l}\text { large majority received } \\
\mathrm{BL} / 2 \text { weeks to } 7 \text { years/ } \\
\text { variable }\end{array}$ & $\begin{array}{l}\text { various } \\
\text { medications } \\
\text { in } 8 \text { patients }\end{array}$ & $\begin{array}{l}\text { Reduced number of hospitalizations for all patients relative } \\
\text { to comparative period (of unspecified length) w/o C-ECT. } 4 \\
\text { patients continued to receive CECT at the time of publica- } \\
\text { tion; C-ECT stopped in } 1 \text { patient due to nonresponse, } 1 \text { due } \\
\text { to memory problems, } 5 \text { due to patient preference, and } 1 \text { due } \\
\text { to PVCs and arrhythmia. }\end{array}$ \\
\hline $\begin{array}{l}\text { Tsao } \\
\text { et al. [46] } \\
(2004)\end{array}$ & case report & $\begin{array}{l}\mathrm{BD} \text {, manic } \\
\text { episode }\end{array}$ & 1 & $\begin{array}{l}\mathrm{BL} />4 \text { months/every } 2 \\
\text { weeks }\end{array}$ & unspecified & $\begin{array}{l}\text { C-ECT effective in maintaining euthymia after resolution of } \\
\text { acute manic episodes. C-ECT stopped due to patient prefer- } \\
\text { ence. Relapse into mania } 1 \text { month later, ECT re-started. }\end{array}$ \\
\hline $\begin{array}{l}\text { Wijkstra } \\
\text { and Nolen } \\
{[47](2005)}\end{array}$ & case report & $\begin{array}{l}\text { MDD, } \\
\text { w/psychotic } \\
\text { features }\end{array}$ & 1 & $\begin{array}{l}\text { BL/6 years } 2 \text { months/ } \\
\text { variable (range: weekly - } \\
\text { monthly) }\end{array}$ & $\begin{array}{l}\text { oxazepam } \\
(20 \mathrm{mg} / \mathrm{d}) \text { or } \\
\text { zolpidem } \\
(7.5 \mathrm{mg} / \mathrm{d})\end{array}$ & $\begin{array}{l}\text { C-ECT effective (HRSD } 17<10 \text { at } 66 \text { of } 70 \text { assessments, }<5 \text { at } \\
55 \text { of } 70 \text { assessments), reduced number of hospitalizations } \\
\text { compared to } 4 \text {-year pre-C-ECT period, no cognitive deterio- } \\
\text { ration as measured by patient report and MMSE. }\end{array}$ \\
\hline
\end{tabular}


Table 1 (continued)

\begin{tabular}{|c|c|c|c|c|c|c|}
\hline Author & Design & $\begin{array}{l}\text { Diagnostic } \\
\text { criteria }\end{array}$ & Subjects, $\mathrm{n}$ & $\begin{array}{l}\text { C-ECT type/ } \\
\text { duration/interval }\end{array}$ & Medication & Outcome/comments \\
\hline $\begin{array}{l}\text { Abraham } \\
\text { et al. [48] } \\
(2006)\end{array}$ & $\begin{array}{l}\text { retrospective } \\
\text { review }\end{array}$ & depression & 18 & $\begin{array}{l}\text { BF/10.8 months (mean); } \\
8.0 \text { months }(\mathrm{SD}) / 2.6 \text { weeks } \\
\text { (mean) }\end{array}$ & $\begin{array}{l}\text { various } \\
\text { medications }\end{array}$ & $\begin{array}{l}11 \% \text { relapse rate, relapse rate higher for patients who were } \\
\text { more symptomatic at the end of acute ECT. Severe memory } \\
\text { impairment in } 5.7 \% \text { as measured by notes in record. }\end{array}$ \\
\hline $\begin{array}{l}\text { Suzuki } \\
\text { et al. [49] } \\
(2006)\end{array}$ & case report & $\begin{array}{l}\text { MDD, with } \\
\text { psychotic } \\
\text { features }\end{array}$ & 1 & $\begin{array}{l}\text { BL/4 years/variable ( } 4 \\
\text { weekly, } 10 \text { every } 2 \text { weeks, } \\
6 \text { every } 3 \text { weeks, } 34 \text { every } \\
4 \text { weeks) }\end{array}$ & $\begin{array}{l}\text { milnacipran } \\
(75 \mathrm{mg} / \mathrm{d})\end{array}$ & $\begin{array}{l}\text { Age } 70 . \text { Residual symptoms improved (acute phase end } \\
\text { HRSD }_{17}=11 \text { to HRSD } \\
\text { remained remitted }\left(\mathrm{HRSD}_{17}=3 \text { after } 2 \text { years of Cerebral hypoperfusion re- }\right. \\
\text { solved, no adverse effects }(\text { acute phase baseline } \mathrm{MMSE}=18 \text {; } \\
\text { after } 2.5 \text { years } \mathrm{MMSE}=25) \text {. }\end{array}$ \\
\hline $\begin{array}{l}\text { Sienaert } \\
\text { and } \\
\text { Peuskens } \\
{[50]} \\
(2006)\end{array}$ & case report & $\begin{array}{l}\text { BD, mixed } \\
\text { episode }\end{array}$ & 1 & RUL/37 months/variable & $\begin{array}{l}\text { haloperidol } \\
(5 \mathrm{mg} / \mathrm{d})\end{array}$ & $\begin{array}{l}\text { C-ECT effective in preventing mixed episodes. Cognitive de- } \\
\text { terioration associated with depressive episode improved and } \\
\text { there were no signs of retrograde amnesia as measured by NP } \\
\text { battery. 'C-ECT was stopped because of an increasing anes- } \\
\text { thetic risk due to morbid obesity.' Patient relapsed } 3 \text { months } \\
\text { after C-ECT was discontinued. }\end{array}$ \\
\hline $\begin{array}{l}\text { Nascimento } \\
\text { et al., } \\
(2006)[51]\end{array}$ & case report & $\begin{array}{l}\text { BD, manic } \\
\text { episode }\end{array}$ & 1 & $\mathrm{BT} / 16$ months/variable & $\begin{array}{l}\text { no } \\
\text { medication }\end{array}$ & $\begin{array}{l}\text { Age } 45 \text {. No manic or depressive episodes, MMSE scores } \\
\text { above } 29 \text {, no subjective treatment-related complaints. }\end{array}$ \\
\hline $\begin{array}{l}\text { Yero } \\
\text { et al. [52] } \\
(2006)\end{array}$ & case report & BD, PSAS & 2 & $\begin{array}{l}\mathrm{BT} \text { and } \mathrm{BF} / 9 \text { months and } \\
\text { unknown/variable }\end{array}$ & $\begin{array}{l}\text { valproic acid } \\
\text { and paroxetine }\end{array}$ & $\begin{array}{l}\text { Both patients experienced remission from mood disorder } \\
\text { and PSAS. }\end{array}$ \\
\hline $\begin{array}{l}\text { Balke and } \\
\text { Varma [53] } \\
(2007)\end{array}$ & case report & $\begin{array}{l}\text { MDD and } \\
\text { possible PD }\end{array}$ & 1 & $\begin{array}{l}\text { unspecified/ } 6 \text { years/ } \\
\text { monthly and bimonthly }\end{array}$ & unspecified & $\begin{array}{l}\text { Age } 78 \text {. Long-term C-ECT effective and did not cause cogni- } \\
\text { tive impairment (MMSE score range } 25 / 30 \text { to } 28 / 30 \text { ). 'Over } \\
\text { the years, successful maintenances ECT has helped her to } \\
\text { continue to live independently ...' }\end{array}$ \\
\hline $\begin{array}{l}\text { Bozkurt } \\
\text { et al. }[54] \\
(2007)\end{array}$ & case report & $\begin{array}{l}\text { psychotic } \\
\text { depression in } \\
\text { a pregnant } \\
\text { patient }\end{array}$ & 1 & $\begin{array}{l}\text { BL/15 weeks/variable ( } 3 \\
\text { weekly, } 3 \text { monthly) }\end{array}$ & $\begin{array}{l}\text { no } \\
\text { medication }\end{array}$ & $\begin{array}{l}\text { C-ECT effective (HSRD = } 3 \text { after } 3 \text { weekly C-ECT), no com- } \\
\text { plications except for pelvic pain and transient fetal arrhyth- } \\
\text { mias. }\end{array}$ \\
\hline $\begin{array}{l}\text { Zisselman } \\
\text { et al. [55] } \\
(2007)\end{array}$ & case report & $\begin{array}{l}\text { MDD } \\
\text { w/psychotic } \\
\text { features, BD } \\
\text { w/moderate } \\
\text { mental } \\
\text { retardation }\end{array}$ & 2 & BL/several years/weekly & $\begin{array}{l}\text { various } \\
\text { medications }\end{array}$ & $\begin{array}{l}\text { Weekly C-ECT for extended periods effective and well toler- } \\
\text { ated, with attempts to increase the interval between treat- } \\
\text { ments unsuccessful. }\end{array}$ \\
\hline $\begin{array}{l}\text { Gupta } \\
\text { et al. [56] } \\
(2008)\end{array}$ & $\begin{array}{l}\text { retrospective } \\
\text { review } \\
\text { w/matched } \\
\text { control group } \\
\text { (acute ECT } \\
\text { w/out C-ECT) }\end{array}$ & MDD & $\begin{array}{l}\mathrm{C}-\mathrm{ECT}=19 \\
\text { no } \\
\mathrm{C}-\mathrm{ECT}=19\end{array}$ & $\begin{array}{l}\text { BL and RUL/range } \\
18-329 \text { weeks } / 2.5 \text { weeks } \\
\text { (mean) }\end{array}$ & unspecified & $\begin{array}{l}\text { C-ECT phase showed within-group reduction in mean ad- } \\
\text { mission rates compared to pre-ECT period (mean } 1.00 \text { ad- } \\
\text { missions/year in } 2 \text {-year pre-ECT period, to } 0.316 \text { admis- } \\
\text { sions/year during C-ECT, to } 0.2555 \text { admissions/year in post- } \\
\text { C-ECT. Bed occupancy was signifycantly lower for C-ECT } \\
\text { compared to control group }(\mathrm{p}<0.001 \text { ). }\end{array}$ \\
\hline $\begin{array}{l}\text { Odeberg } \\
\text { et al. [57] } \\
(2008)\end{array}$ & $\begin{array}{l}\text { retrospective } \\
\text { review }\end{array}$ & $\mathrm{MDD}, \mathrm{BD}$ & 41 & $\begin{array}{l}\mathrm{BL} \text { and } \mathrm{RUL} />4 \text { months/ } \\
\text { variable ( } 2 \text {-week interval } \\
\text { common) }\end{array}$ & $\begin{array}{l}\text { various } \\
\text { medications }\end{array}$ & $\begin{array}{l}\text { Over } 6 \text {-year comparison period ( } 3 \text { years before and after C- } \\
\text { ECT + medication). 'The total number of hospital days was } \\
\text { reduced by } 76 \% \text {, and the numbers of hospitalized patients } \\
\text { and hospitalizations were both reduced by } 64 \% \text {.' }\end{array}$ \\
\hline $\begin{array}{l}\text { O'Connor } \\
\text { et al. [58] } \\
(2009)\end{array}$ & $\begin{array}{l}\text { retrospective } \\
\text { review }\end{array}$ & $\begin{array}{l}\text { elderly } \\
\text { patients, } \\
\text { MDD, BD }\end{array}$ & 54 & $\begin{array}{l}\text { laterality varied/varied/ } \\
\text { varied; '... started with } \\
\text { weekly .... to fortnightly ... } \\
\text { to monthly.' }\end{array}$ & $\begin{array}{l}\text { various } \\
\text { medications }\end{array}$ & $\begin{array}{l}\text { Over } 4 \text {-year comparison period ( } 2 \text { years before and after } \\
\text { C-ECT), number of hospital admissions and length of stay } \\
\text { declined. '.... admissions were halved in number and quar- } \\
\text { tered in duration.' 'Most patients had no recorded adverse } \\
\text { event.' '... } 8 \text { instances of memory loss or confusion ... resolved } \\
\text { safely.' }\end{array}$ \\
\hline
\end{tabular}

MDD = Major depressive disorder; BD = bipolar disorder; PD = Parkinson's disease; RCBD = rapid cycling bipolar disorder; PSAS = persistent sexual arousal syndrome; $\mathrm{BL}=$ bilateral; $\mathrm{BF}=$ bifrontal; RUL = right unilateral; C-PHARM = continuation pharmacotherapy; HRSD = Hamilton rating scale for depression; MMSE = Mini mental state examination; GAF = global assessment of functioning; $\mathrm{PVC}=$ premature ventricular contraction; $\mathrm{SD}=$ standard deviation; $\mathrm{SSMQ}=\mathrm{Squire}$ subjective memory questionnaire. 
Table 2. Prospective studies on the efficacy/effectiveness and tolerability of C-ECT (1998-2009)

\begin{tabular}{|c|c|c|c|c|c|c|}
\hline Author & Design & $\begin{array}{l}\text { Diagnostic } \\
\text { criteria }\end{array}$ & Subjects, n & $\begin{array}{l}\text { C-ECT type/ } \\
\text { duration/interval }\end{array}$ & Medication & Outcome/comments \\
\hline $\begin{array}{l}\text { Wijkstra } \\
\text { et al. [59] } \\
(2000)\end{array}$ & $\begin{array}{l}\text { prospective } \\
\text { naturalistic } \\
\text { study }\end{array}$ & MDD & 12 & $\begin{array}{l}\text { BL/6 months/variable } \\
\text { ('between once/week } \\
\text { and once } / 3-4 \text { weeks) }\end{array}$ & $\begin{array}{l}\text { no } \\
\text { medication }\end{array}$ & $\begin{array}{l}50 \% \text { remained remitted, } \mathrm{HRSD}_{17}<4,6 \text { of } 12 \text { re- } \\
\text { lapsed and required hospitalization. 'No drop- } \\
\text { outs.' }\end{array}$ \\
\hline $\begin{array}{l}\text { Swoboda } \\
\text { et al. [60] } \\
(2001)\end{array}$ & $\begin{array}{l}\text { prospective } \\
\text { comparison } \\
\text { w/matched } \\
\text { pharmacotherapy } \\
\text { control group }\end{array}$ & $\mathrm{MDD}, \mathrm{BD}$ & $\begin{array}{l}13 \\
13\end{array}$ & $\begin{array}{l}\text { BL and unilateral/9.61 } \\
\text { months (mean); } 2-24 \\
\text { months (range)/ } \\
\text { variable, '... usually } \\
\text { weekly ... bi-weekly to } \\
\text { monthly sessions'. }\end{array}$ & $\begin{array}{l}\text { various } \\
\text { pharmacotherapy }\end{array}$ & $\begin{array}{l}\text { Post C-ECT MMSE }=28.52 \text { (mean); control } \\
\text { group MMSE }=27.66 \text { (mean). M-ECT stopped } \\
\text { due to: cognitive-related issues for } 2 \text { patients and } \\
\text { hypertension for } 1 \text { patient. These data include } \\
\text { scores from } 8 \text { patients with SAD. C-ECT + medi- } \\
\text { cation reduced hospitalization by } 77 \% \text { com- } \\
\text { pared to } 46 \% \text { with pharmacotherapy alone, at } 12 \\
\text { months. }\end{array}$ \\
\hline
\end{tabular}

\begin{tabular}{lll}
\hline Datto et al. & $\begin{array}{l}\text { prospective } \\
\text { naturalistic pilot (2001) } \\
\text { study using } \\
\text { telephone } \\
\text { assessments to } \\
\text { detect cognitive }\end{array}$ & $\begin{array}{l}\text { MDD, BD, } \\
\text { organic mood } \\
\text { disorder }\end{array}$ \\
& \\
&
\end{tabular}

BL and RUL/6 months uncontrolled Overall tolerability of ECT supported. 1 patient minimum/2.92 weeks and unspecified experienced cognitive impairment that persisted (mean); 0.966 (SD) pharmacotherapy the day after C-ECT but resolved 1 week later as measured by a telephone cognitive battery. 'One of nine tests (verbal fluency category) showed group level effects, with decrements in performance the day after a treatment.' 'Many of these patients noted the most significant negative effect on their cognition occurs the day of their treatment.'

\begin{tabular}{|c|c|c|c|c|c|}
\hline $\begin{array}{l}\text { Rami- } \\
\text { Gonzalez } \\
\text { et al. [62] } \\
(2003)\end{array}$ & $\begin{array}{l}\text { prospective } \\
\text { matched } \\
\text { comparison }\end{array}$ & $\begin{array}{l}\text { MDD, in } \\
\text { remission } \\
\text { for at least } \\
3 \text { months }\end{array}$ & $\begin{array}{l}\text { C-ECT = } 11 \\
\text { C-PHARM = } 11\end{array}$ & $\begin{array}{l}\text { BL/27.2 months } \\
\text { (mean); } 17.7(\mathrm{SD}) / \\
52.7 \text { days (mean); } \\
16.8 \text { (SD) }\end{array}$ & $\begin{array}{l}\text { various } \\
\text { medications } \\
\text { (in both groups) }\end{array}$ \\
\hline
\end{tabular}

C-ECT frontal function significantly impaired on $4 / 5$ tests compared to depressed controls who never had ECT. C-ECT short-term memory scores lower; long-term memory characterized as normal. C-ECT MMSE = 27.5 (mean) $/$ HRSD = 3.5 (mean); C-PHARM MMSE = 28.2 (mean); $\mathrm{HRSD}=2.5$ (mean).

\begin{tabular}{|c|c|c|c|c|}
\hline $\begin{array}{l}\text { Vothknecht } \\
\text { et al. [63] } \\
(2003)\end{array}$ & $\begin{array}{l}\text { prospective } \\
\text { naturalistic } \\
\text { comparison }\end{array}$ & $\mathrm{MDD}, \mathrm{BD}$ & $\begin{array}{l}\text { C-ECT }=9 \\
\text { C-PHARM = } 13\end{array}$ & $\begin{array}{l}\text { BL and RUL/65 weeks } \\
\text { (mean); } 16-161 \\
\text { (range)/2.2 weeks } \\
\text { (mean); } 0.9-4.4 \\
\text { (range) }\end{array}$ \\
\hline
\end{tabular}

'psychotropic

medication was

discontinued if

possible' for

C-ECT group

mixed pharma-

cotherapy in

Following acute ECT and then 6 months of continuation treatment, C-ECT cognition comparable to C-PHARM as measured by NP battery. After 6 months, 'cognitive function remained stable throughout' C-ECT 'with an average duration of 65 weeks'. Depression scores stabilized in both groups, with a trend to further improvement in C-PHARM group C-ECT group. At end of follow-up period, more patients in C-PHARM relapsed compared to CECT.

\begin{tabular}{|c|c|c|c|c|}
\hline $\begin{array}{l}\text { Rami et al. } \\
{[64]} \\
(2004)\end{array}$ & $\begin{array}{l}\text { prospective } \\
\text { comparison }\end{array}$ & MDD, BD & $\begin{array}{l}\text { C-ECT }=14 \\
\text { C-PHARM } \\
\text { control }=8\end{array}$ & $\begin{array}{l}\text { BL/1 year/36.85 days } \\
\text { (mean); } 14.41(\mathrm{SD}) ; \\
15-60 \text { days (range) }\end{array}$ \\
\hline
\end{tabular}

all C-ECT

patients also had

C-PHARM of unspecified type

C-PHARM

medication

unspecified

Cognitive tolerability of M-ECT supported as measured by NP battery and compared to CPHARM control group (no ECT). Baseline CECT means: HRSD $=3.8 ;$ MMSE $=28.0 ; 1$ year $\mathrm{M}-\mathrm{ECT}$ retest means: $\mathrm{HRSD}=3.2$; $\mathrm{MMSE}=$ 28.90 .

C-ECT:

lorazepam and

After acute ECT, similar efficacy and tolerability

Kellner et al. randomized

MDD

$\mathrm{C}-\mathrm{ECT}=89$

BT/6 months/fixed

[65] (2006) controlled trial

$\begin{array}{ll}\text { C-ECT }=89 & \text { BT/6 months/fixed } \\ \text { C-PHARM }=95 & \text { schedule }(4 \text { weekly, } \\ & 8 \text { biweekly, } \\ & 2 \text { monthly })\end{array}$

diphenhydramine Relapse rates $37.1 \%$ for $\mathrm{C}-\mathrm{ECT} ; 31.6 \%$ for as needed in both PHARM. Drop-out rates $16.8 \% \mathrm{C}$-ECT; $22.1 \% \mathrm{C}$ arms C-PHARM: $\mathrm{Li}+$ measured by the MMSE. nortriptyline

\begin{tabular}{|c|c|c|c|c|c|}
\hline $\begin{array}{l}\text { Odeberg } \\
\text { et al. [57] } \\
(2008)\end{array}$ & $\begin{array}{l}\text { partial prospective } \mathrm{MDD}, \mathrm{BD} \\
\text { naturalistic follow- } \\
\text { up (following } \\
\text { retrospective } \\
\text { review, see table 1) }\end{array}$ & 16 & $\begin{array}{l}\text { BL and RUL/ } \\
>4 \text { months/variable } \\
\text { (2-week interval } \\
\text { common) }\end{array}$ & $\begin{array}{l}\text { various } \\
\text { medications }\end{array}$ & $\begin{array}{l}\text { Subset of patients assessed at follow-up interview } \\
\text { mean time-point: } 15 \text { months; range: } 4-29 \text { months } \\
\text { and } 87 \% \text { were in remission (defined as MADRS } \\
<10 \& \text { CGI <2). C-ECT ongoing in } 56 \% \text { of pa- } \\
\text { tients. }\end{array}$ \\
\hline
\end{tabular}


Table 2 (continued)

\begin{tabular}{|c|c|c|c|c|c|c|}
\hline Author & Design & $\begin{array}{l}\text { Diagnostic } \\
\text { criteria }\end{array}$ & Subjects, n & $\begin{array}{l}\text { C-ECT type/ } \\
\text { duration/interval }\end{array}$ & Medication & Outcome/comments \\
\hline $\begin{array}{l}\text { Navarro } \\
\text { et al. [66] } \\
(2008)\end{array}$ & $\begin{array}{l}\text { randomized } \\
\text { controlled trial }\end{array}$ & $\begin{array}{l}\text { elderly } \\
\text { patients, } \\
\text { MDD } \\
\text { w/psychotic } \\
\text { features }\end{array}$ & $\begin{array}{l}\text { C-ECT = } 16 \\
\text { C-PHARM = } 17\end{array}$ & $\begin{array}{l}\text { BL/2 years weekly for } \\
4 \text { weeks, every } 2 \text { weeks } \\
\text { for } 1 \text { month, monthly }\end{array}$ & $\begin{array}{l}\text { nortriptyline/ } \\
\text { nortriptyline and } \\
\text { risperidone for } \\
10 \text { weeks, then } \\
\text { nortriptyline } \\
\text { alone }\end{array}$ & $\begin{array}{l}\text { After acute ECT + nortriptyline, 'the risk of re- } \\
\text { lapse/recurrence was } 8 \text {-fold higher during the first } \\
\text { year of follow-up in the patient subgroup treated } \\
\text { w/o C-ECT.' 'No significant differences were } \\
\text { found in the subscores on the UKU, in the UKU } \\
\text { global score, or in mean changes in ECG intervals, } \\
\text { heart rate, or diastolic and systolic blood pres- } \\
\text { sure.' Changes in MMSE score also not signifi- } \\
\text { cant. }\end{array}$ \\
\hline
\end{tabular}

$\mathrm{MDD}=$ Major depressive disorder; $\mathrm{BD}=$ bipolar disorder; $\mathrm{BL}=$ bilateral; $\mathrm{RUL}=$ right unilateral; $\mathrm{C}-\mathrm{PHARM}=$ continuation pharmacotherapy; $\mathrm{HRSD}=$ Hamilton rating scale for depression; MMSE = Mini mental state examination; CGI = Clinical global impression; NP = neuropsychological; MADRS = Montgomery-Åsberg depression rating scale; UKU = Udvalg for kliniske undersøgelser side effect rating scale; SAD = schizoaffective disorder; SD = standard deviation.

monotherapy $(60 \%)$ and placebo $(84 \%)$. There were no memory outcome differences between unrelapsed recipients of C-ECT and C-PHARM at six months as determined by an extensive neuropsychological battery [67]. The authors concluded that memory effects should have only a small role in the choice between C-ECT and CPHARM. These results confirmed the value of C-ECT for relapse prevention in patients with depression successfully treated with ECT. However, with over one-third of patients in even the most effective arm of both RCTs relapsing during the 6 months of continuation treatment, it is clear that neither C-ECT nor combination pharmacotherapy alone represents the last word on post-acute-ECT prolongation of remission $[11,65]$.

In an effort to improve the rates of sustained remission after completion of a successful acute course of ECT, clinicians and investigators are turning to more intensive follow-up interventions. For example, in a relatively small prospective, single-blind study, Navarro et al. [66] randomized 33 elderly patients with psychotic depression responsive to an acute course of ECT to receive either CECT plus nortriptyline, or nortriptyline alone, both arms combined with risperidone, for 10 weeks. They report that after successful acute ECT, 'the risk of relapse/recurrence was eightfold higher during the first year of followup in the patient subgroup treated without C-ECT'. They also report no differences in tolerability between groups.

As listed in table 1, a growing number of uncontrolled studies report positive outcomes using the combination of C-ECT and medications. Thus, the reasonable next step in C-ECT research is to test in a controlled fashion whether the combination of C-ECT and C-PHARM may improve remission rates beyond those seen with either intervention alone. For example, the CORE group has embarked on a multicenter study, Prolonging Remission in Depressed Elderly (PRIDE), to test this hypothesis. This study will compare, over a 6-month period, the relative efficacy of lithium and venlafaxine versus lithium, venlafaxine, and continuation ECT in geriatric depression.

\section{C-ECT/M-ECT Frequency and Duration}

We lack established guidelines with regard to frequency and duration of C-ECT. The guidelines used for continuation pharmacotherapies, as well as common sense, would call for a duration of C-ECT at least equal to the expected natural course of the episode of illness. In the case of major depression and bipolar disorder, this duration should be at least 8-20 weeks. Typically, C-ECT courses for depression last from 2 to 6 months. Longer courses of treatment and M-ECT are indicated for patients with more severe, recurrent and treatment-resistant forms of depression. In these cases, M-ECT should be administered at the minimum frequency necessary to prevent relapse. The APA Task Force calls for reevaluation of the necessity of treatment at least every 6 months, taking into consideration both beneficial and adverse effects.

In clinical practice, psychiatrists often follow one of three types of schedules: (1) a tapered schedule that usually starts with weekly treatments for 2-4 weeks, followed by a gradual decrease in the frequency to once per month, 
(2) a fixed-interval schedule with treatments every 1-4 weeks, and (3) an 'as-needed' approach with 1-2 treatments each time there are signs of relapse $[18,19]$.

However, the question of optimum frequency and duration of C-ECT has not been studied systematically. The PRIDE study C-ECT arm features an initial fixed, tapered treatment schedule (with 4 ECT treatments in the first month) followed by a treatment algorithm based on individual patient symptomatology [68]. Patients are given 0,1 , or 2 treatments each week based on a comprehensive algorithm that takes into account changes in the Hamilton Rating Scale for Depression - 24 items $\left(\mathrm{HRSD}_{24}\right)$ scores at the previous and current assessments, and readjusts for baseline $\mathrm{HSRD}_{24}$ score. This approach seeks to treat residual symptoms early in the continuation treatment course and to treat the reemergence of symptoms before full syndromal relapse, in order to improve long-term outcome.

\section{Risks}

The risks of C-ECT are similar to those of the index treatment. It should be noted that most C-ECT treatments are given in an outpatient setting and thus may only be appropriate for certain patients. This situation creates the need for very cooperative and reliable patients, who will follow the 'nothing by mouth after midnight' instructions, and who have a strong support system that will provide care and transportation after each treatment. Patients should not be allowed to drive home nor for $24 \mathrm{~h}$ after a treatment.

Some patients (especially the elderly) may remain confused for longer periods after ECT. In such cases, the risk of falls is substantial. Caregivers should be educated about this specific risk, and provide adequate supervision.

As C-ECT treatments are given less frequently than in the index course, cognitive difficulties are shorter lasting and not cumulative as in index courses. Several cases of patients who received many treatments over years without problems have been reported. In an earlier report by Barnes et al. [69], a 74-year-old patient who received more than 400 treatments did not show any signs of progressive cognitive deterioration. In the CORE C-ECT study, patients who received C-ECT did not differ in cognitive side effects from the C-PHARM group. The drop-out rates due to treatment side effects was slightly higher for the medication group (21-17\%), but the difference did not reach statistical significance [65].

\section{Technical Issues}

\section{Anesthesia}

The same treatment techniques as used in the index course are applied in C-ECT. Anesthetic medications and dosages remain the same, unless a change in the patient's general health or the use of concurrent medications dictates a different approach. Posttreatment evaluation includes assessment of orientation, alertness, gait and vital signs. When reoriented, with stable gait and vital signs, patients are allowed to leave the treatment facility.

\section{Concurrent Medications}

The efficacy of combination C-ECT and antidepressant medications for relapse prevention in mood disorders has not been studied systematically. However, nearly all of the published literature on C-ECT cites the use of combined C-ECT with medications. Navarro et al. [66] combined nortriptyline with acute and continuation ECT in a sample of elderly depressed patients, and found the combination to be safe and effective in decreasing post-acute ECT relapse.

Safety concerns are the same in any ECT course. Some clinicians advise patients to discontinue all psychotropic medications other than antipsychotic agents, 1-2 days prior to each C-ECT and then continue with regular dosing after treatment [70]. However, a recent study [71] reports the safe use of nortriptyline or venlafaxine during an acute course of ECT. Interestingly, concurrent pharmacotherapy enhanced efficacy rates up to $15 \%$ compared to ECT alone in that study.

The role of concurrent use of mood stabilizers and CECT is also unknown. Caution should be used when lithium is prescribed in parallel with C-ECT because of the reported increased risk of confusion and cognitive impairment [70]. It is recommended that serum levels be kept lower than usual full therapeutic concentrations at the time of ECT. Patients should be instructed not to take any lithium the day before and the morning of ECT. Anticonvulsants should be avoided as they increase the seizure threshold and may make the elicitation of a seizure during ECT difficult. Benzodiazepines may interfere with the efficacy of the treatment [72]. Practitioners should consider the use of shorter-acting benzodiazepines such as lorazepam, or reversing benzodiazepines with flumazenil at the time of ECT [73].

Systemic medications should be continued. Specific attention should be given to concurrent use and (possible dosage changes) of nonpsychiatric medications that may affect treatment parameters, e.g. seizure duration or sei- 
zure threshold. Agents such as $\beta$-blockers and lidocaine may interfere with seizure induction or duration, while calcium-channel blockers, antiepileptics and some analgesics may increase seizure threshold. Theophylline has been associated with prolonged seizures [70]. Insulin-dependent patients may need dosage and time of dosage adjustments on the days of treatment.

\section{Electrode Placement and Electrical Dosage}

The optimum electrode placement for C-ECT has not been studied. Most studies report bilateral (bifrontotemporal) placement, but some use right unilateral placement. There are no studies comparing the efficacy and safety of different placements in the continuation and maintenance phases. Commonly, the technique that was effective in the index course is used in C-ECT or M-ECT [74]. It is noteworthy that concerns about cognitive impairment that may have guided the choice of electrode placement during the index course may not be as relevant during C-ECT. Since the treatments during C-ECT are given less frequently, there is usually ample time between treatments to allow for cognitive recovery. The CORE CECT study, which was performed with bilateral ECT during both index and continuation phases, showed no major differences between the ECT group and the pharmacotherapy group in outcome or adverse effects, thus suggesting that bilateral ECT is a viable option in C-ECT.

Optimal electrical doses for use during C-ECT have also not been studied. Because of the infrequent schedule, the seizure threshold changes little or decreases between treatments, in contrast to the rise in seizure threshold typically observed during an index course of ECT. Clinicians should try to adjust electrical dosages to avoid unnecessary overstimulation and unwanted cognitive side effects.

\section{Interim Evaluations}

No specific examinations are required before the initiation of a C-ECT course, provided that these were performed before the onset of the index course. However, given the fact that outpatients are not under continuous direct medical observation between treatments, an interval psychiatric and medical history should be obtained before each treatment. Physical complaints and findings, especially those pertaining to systems at risk with ECT (i.e. cardiovascular, central nervous and musculoskeletal systems), should be evaluated before each treatment.

The APA Task Force and the ACT Task Force on ambulatory ECT recommend the performance of a physical examination, laboratory tests (including hematocrit, he-

C-ECT and M-ECT moglobin and serum electrolytes) every 6 months in patients undergoing C-ECT and M-ECT [3, 18].

\section{Special Instructions}

Patients should be instructed not to eat or drink anything for at least $8 \mathrm{~h}$ prior to each treatment. Although reliable outpatients may come for treatment alone, a family member or other reliable caregiver should take them home. Adequate supervision should be provided for several hours after treatment and for longer periods if there is a prior history of prolonged postictal confusion. Some patients may need to miss a day of work. It is prudent that instructions are given to the patients and caregivers in writing.

\section{Consent}

A new informed-consent form should be signed before the beginning of C-ECT. This consent form should reflect the particularities of the treatment and should be renewed according to state and local laws and institutional policies. When such laws and policies are vague, it is recommended that renewal of consent be done at least every 12 treatments or 6 months, whichever occurs first [3].

\section{Cost}

In an article not included in the tables, Aziz et al. [75] conducted a cost-utility analysis of M-ECT compared to maintenance pharmacotherapy in depressed elderly patients. This sophisticated analysis took both objective and subjective reports of the disease into consideration, and adjusted for quantitative (number of years) and qualitative (quality of years) factors. These authors found that M-ECT yielded a significantly higher number of 'quality-adjusted life years' compared to C-PHARM (11.43 vs. 7.55 , respectively), and at much lower cost per year (USD24,616 vs. 57,762). Other published reports support ECT as a cost-effective treatment modality in the continuation and maintenance phases $[35,76]$.

\section{Conclusions}

C-ECT and M-ECT are valuable treatment modalities to prevent relapse and recurrence of mood disorders in patients who have responded to an index course of ECT. $\mathrm{C}$-ECT and M-ECT are underused and insufficiently studied despite positive clinical experience of more than 70 years.

Neuropsychobiology 2011;64:129-140 
There are now adequate controlled data to conclude that without active treatment following an acute course of ECT, the short-term relapse rate is unacceptably high. It is still unknown whether there are features of the underlying disorder or the initial ECT course that influence the relapse rate or the response to subsequent continuation and maintenance treatment.

As we have shown in this review, both medications (particularly the combination of antidepressants and mood stabilizers) and ECT are effective as continuation and maintenance interventions following ECT-induced remission. Further research is necessary to identify which approach is better for a given individual, and to explore methods of enhancing the still inadequate protection against relapse provided by either option. For example, current research is seeking to assess the role of combined antidepressant pharmacotherapy plus ECT in both acute and continuation phases of treatment. Individualized CECT and M-ECT schedules are also under study, using the patient's weekly depression rating to determine the number (if any) of treatments to be delivered during the maintenance phase [68].

Thus, studies that are currently under way should allow more definitive recommendations regarding the choice, frequency and duration of continuation and maintenance treatment following acute ECT. This knowledge will enable the field to move closer to the goals of personalizing treatment and reducing the burden of depression and other serious mental disorders.

\section{References}

1 Abrams R: Electroconvulsive therapy, ed 2. Oxford, Oxford University Press, 1992.

$>2$ Rohland BM, Carroll BT, Jacoby RG: ECT in the treatment of the catatonic syndrome. J Affect Disord 1993;29:255-261.

3 American Psychiatric Association: Task Force on Electroconvulsive Therapy. The Practice of Electroconvulsive Therapy: Recommendations for Treatment, Training, and Privileging. Washington, American Psychiatric Association, 2001.

-4 Petrides G, Fink M, Husain MM, Knapp RG, Rush AJ, Mueller M, Rummans TA, O’Connor KM, Rasmussen KG Jr, Bernstein HJ, Biggs M, Bailine SH, Kellner CH: ECT remission rates in psychotic versus nonpsychotic depressed patients: a report from CORE. J ECT 2001;17:244-253.

$>5$ Birkenhager TK, van den Broek WW, Mulder PG, de Lely A: One-year outcome of psychotic depression after successful electroconvulsive therapy. J ECT 2005;21:221-226.

6 Kellner CH, Fink M, Knapp R, Petrides G, Husain M, Rummans T, Mueller M, Bernstein H, Rasmussen K, O’Connor K, Smith G, Rush AJ, Biggs M, McClintock S, Bailine S, Malur C: Relief of expressed suicidal intent by ECT: a Consortium for Research in ECT study. Am J Psychiatry 2005;162:977-982.

$>7$ Small JG, Klapper MH, Kellams JJ, Miller MJ, Milstein V, Sharpley PH, Small IF: Electroconvulsive treatment compared with lithium in the management of manic states. Arch Gen Psychiatry 1988;45:727-732.

8 Mukherjee S, Sackeim HA, Schnur DB: Electroconvulsive therapy of acute manic episodes: a review of 50 years' experience [see comment]. Am J Psychiatry 1994;151:169176.
$>9$ Valenti M, Benabarre A, Garcia-Amador M, Molina O, Bernardo M, Vieta E: Electroconvulsive therapy in the treatment of mixed states in bipolar disorder. Eur Psychiatry 2008;23:53-56.

10 American Psychiatric Association: American Psychiatric Association Practice Guide lines for the Treatment of Psychiatric Disorders: Compendium 2006. Arlington, American Psychiatric Publishing, Inc., 2006.

11 Sackeim HA, Haskett RF, Mulsant BH, Thase ME, Mann JJ, Pettinati HM, Greenberg RM, Crowe RR, Cooper TB, Prudic J: Continuation pharmacotherapy in the prevention of relapse following electroconvulsive therapy: a randomized controlled trial. JAMA 2001;285:1299-1307.

12 Kalinowsky LB: Electric convulsive therapy, with emphasis on importance of adequate treatment. Arch Neurol Psychiatry 1943;50: 652-660.

13 Kerman EF: Electroshock therapy: with special reference to relapses and an effort to prevent them. J Nerv Mental Dis 1945;102:231242.

14 Fink M: Impact of the antipsychiatry movement on the revival of electroconvulsive therapy in the United States. Psychiatr Clin North Am 1991;14:793-801.

15 American Psychiatric Association: Electroconvulsive therapy. Report of the task force on electroconvulsive therapy of the American Psychiatric Association. Washington DC, American Psychiatric Association, 1978.

16 Kramer BA: Maintenance ECT: A survey of practice (1986). Convuls Ther 1987;3:260268.
17 American Psychiatric Association: Task Force on Electroconvulsive Therapy. The practice of electroconvulsive therapy: Recommendations for Treatment, Training, and Privileging. Washington, American Psychiatric Association, 1990.

18 Fink M, Abrams R, Bailine S, Jaffe R: Ambulatory electroconvulsive therapy: report of a task force of the association for convulsive therapy. Association for convulsive therapy. Convuls Ther 1996;12:42-55.

19 Petrides G: Continuation ECT: a review. Psychiatric Ann 1998;28:517-523.

20 Monroe RR Jr: Maintenance electroconvulsive therapy. Psychiatr Clin North Am 1991; 14:947-960.

21 National Institute for Clinical Excellence (NICE): Guidance on the Use of Electroconvulsive Therapy (Technology Appraisal 59). NICE, London, 2003.

22 Freeman C: Editorial for ECT. J ECT 2006; 22:1-3.

23 Cole C, Tobiansky R: Electroconvulsive therapy: NICE guidance may deny many patients treatment that they might benefit from. BMJ 2003;327:621.

24 Procopio M: Nice guidelines and maintenance ECT. Br J Psychiatry 2003; 183:263.

-25 Frederikse M, Petrides G, Kellner C: Continuation and maintenance electroconvulsive therapy for the treatment of depressive illness: a response to the National Institute for Clinical Excellence report. J ECT 2006;22: 13-17.

-26 Chanpattana W, Chakrabhand ML, Sackeim HA, Kitaroonchai W, Kongsakon R, Techakasem P, Buppanharun W, Tuntirungsee Y, Kirdcharoen N: Continuation ECT in treatment-resistant schizophrenia: a controlled study. J ECT 1999;15:178-192. 
27 Chanpattana W, Kramer BA: Acute and maintenance ECT with flupenthixol in refractory schizophrenia: sustained improvements in psychopathology, quality of life, and social outcomes. Schizophr Res 2003;63: 189-193.

-28 Rami L, Bernardo M, Valdes M, Boget T, Portella MJ, Ferrer J, Salamero M: Absence of additional cognitive impairment in schizophrenia patients during maintenance electroconvulsive therapy. Schizophr Bull 2004; 30:185-189.

29 Suzuki K, Awata S, Takano T, Ebina Y, Iwasaki $\mathrm{H}$, Matsuoka $\mathrm{H}$ : Continuation electroconvulsive therapy for relapse prevention in middle-aged and elderly patients with intractable catatonic schizophrenia. Psychiatry Clin Neurosci 2005;59:481-489.

- 30 Suzuki K, Awata S, Takano T, Ebina Y, Shindo T, Harada N, Matsuoka H: Adjusting the frequency of continuation and maintenance electroconvulsive therapy to prevent relapse of catatonic schizophrenia in middle-aged and elderly patients who are relapse-prone. Psychiatry Clin Neurosci 2006;60:486-492.

-31 Shimizu E, Imai M, Fujisaki M, Shinoda N, Handa S, Watanabe H, Nakazato M, Hashimoto K, Iyo M: Maintenance electroconvulsive therapy (ECT) for treatment-resistant disorganized schizophrenia. Prog Neuropsychopharmacol Biol Psychiatry 2007;31: 571-573.

- 32 Hustig H, Onilov R: ECT rekindles pharmacological response in schizophrenia. Eur Psychiatry 2009;24:521-525.

-33 Wengel SP, Burke WJ, Pfeiffer RF, Roccaforte WH, Paige SR: Maintenance electroconvulsive therapy for intractable Parkinson's disease. Am J Geriatr Psychiatry 1998;6:263269.

-34 Wachtel LE, Contrucci-Kuhn SA, Griffin M, Thompson A, Dhossche DM, Reti IM: ECT for self-injury in an autistic boy. Eur Child Adolesc Psychiatry 2009;18:458-463.

35 Bonds C, Frye MA, Coudreaut MF, Cunningham $M$, Spearing $M$, McGuire $M$, Guze B: Cost reduction with maintenance ECT in refractory bipolar disorder. J ECT 1998;14: $36-41$.

- 36 Gupta S, Austin R, Devanand D: Lithium and maintenance electroconvulsive therapy. J ECT 1998;14:241-244.

37 Kramer BA: A seasonal schedule for maintenance ECT. J ECT 1999a;15:226-231.

38 Kramer BA: A naturalistic review of maintenance ECT at a university setting. J ECT 1999b; 15:262-269.

- 39 Gagne GG Jr, Furman MJ, Carpenter LL, Price LH: Efficacy of continuation ECT and antidepressant drugs compared to long-term antidepressants alone in depressed patients [see comment]. Am J Psychiatry 2000;157: 1960-1965.

40 Chanpattana W: Combined ECT and clozapine in treatment-resistant mania. J ECT 2000;16:204-207.
41 Stewart JT: Lithium and maintenance ECT. J ECT 2000;16:300-301.

42 Fox HA: Extended continuation and maintenance ECT for long-lasting episodes of major depression. J ECT 2001;17:60-64.

43 Kho KH: Treatment of rapid cycling bipolar disorder in the acute and maintenance phase with ECT. J ECT 2002;18:159-161.

44 Russell JC, Rasmussen KG, O’Connor MK, Copeman CA, Ryan DA, Rummans TA: Long-term maintenance ECT: a retrospective review of efficacy and cognitive outcome. J ECT 2003;19:4-9.

45 Vaidya NA, Mahableshwarkar AR, Shahid $\mathrm{R}$ : Continuation and maintenance ECT in treatment-resistant bipolar disorder. J ECT 2003;19:10-16.

-46 Tsao CI, Jain S, Gibson RH, Guedet PJ, Lehrmann JA: Maintenance ECT for recurrent medication-refractory mania. J ECT 2004; 20:118-119.

47 Wijkstra J, Nolen WA: Successful maintenance electroconvulsive therapy for more than seven years. J ECT 2005;21:171-173.

-48 Abraham G, Milev R, Delva N, Zaheer J: Clinical outcome and memory function with maintenance electroconvulsive therapy: a retrospective study. J ECT 2006;22:43-45.

49 Suzuki K, Awata S, Takano T, Ebina Y, Numata S, Saito A, Ito K, Matsuoka H: Resolution of SPECT-determined anterior cerebral hypoperfusion correlated with maintenance ECT-derived improvement in residual symptoms in a case of late-life psychotic depression. Clin Nucl Med 2006;31:253-255.

50 Sienaert P, Peuskens J: Electroconvulsive therapy: an effective therapy of medicationresistant bipolar disorder [see comment]. Bipolar Disord 2006;8:304-306.

-51 Nascimento AL, Appolinario JC, Segenreich D, Cavalcanti MT, Brasil MA: Maintenance electroconvulsive therapy for recurrent refractory mania [see comment]. Bipolar Disord 2006;8:301-303.

52 Yero SA, McKinney T, Petrides G, Goldstein I, Kellner CH: Successful use of electroconvulsive therapy in 2 cases of persistent sexual arousal syndrome and bipolar disorder. J ECT 2006;22:274-275.

53 Balke LD, Varma A: A case of long-term maintenance ECT in a 78-year-old with depression and possible Parkinson's disease. CNS Spectr 2007;12:325-326.

54 Bozkurt A, Karlidere T, Isintas M, Ozmenler NK, Ozsahin A, Yanarates O: Acute and maintenance electroconvulsive therapy for treatment of psychotic depression in a pregnant patient. J ECT 2007;23:185-187.

55 Zisselman MH, Rosenquist PB, Curlik SM: Long-term weekly continuation electroconvulsive therapy: a case series. J ECT 2007;23: 274-277.

56 Gupta S, Tobiansky R, Bassett P, Warner J: Efficacy of maintenance electroconvulsive therapy in recurrent depression: a naturalistic study. J ECT 2008;24:191-194.
57 Odeberg H, Rodriguez-Silva B, Salander P, Martensson B: Individualized continuation ECT and medication as a bridge to relapse prevention following an index course of ECT in severe mood disorders: a naturalistic 3. year cohort study. J ECT 2008;24:183-190.

58 O'Connor DW, Gardner B, Presnell I, Singh $\mathrm{D}$, Tsanglis $\mathrm{M}$, White E: The effectiveness of continuation-maintenance ECT in reducing depressed older patients' hospital re-admissions. J Affect Disord 2009.

59 Wijkstra J, Nolen WA, Algra A, van Vliet IM, Kahn RS: Relapse prevention in major depressive disorder after successful ECT: a literature review and a naturalistic case series [see comment]. Acta Psychiatr Scand 2000; 102:454-460

60 Swoboda E, Conca A, Konig P, Waanders R, Hansen M: Maintenance electroconvulsive therapy in affective and schizoaffective disorder. Neuropsychobiology 2001;43:23-28.

61 Datto CJ, Levy S, Miller DS, Katz IR: Impact of maintenance ECT on concentration and memory. J ECT 2001;17:170-174.

62 Rami-Gonzalez L, Salamero M, Boget T, Catalan R, Ferrer J, Bernardo M: Pattern of cognitive dysfunction in depressive patients during maintenance electroconvulsive therapy. Psychol Med 2003;33:345-350.

63 Vothknecht S, Kho KH, van Schaick HW, Zwinderman AH, Middelkoop H, Blansjaar BA: Effects of maintenance electroconvulsive therapy on cognitive functions. J ECT 2003;19:151-157.

64 Rami L, Bernardo M, Boget T, Ferrer J, Portella MJ, Gil-Verona JA, Salamero M: Cognitive status of psychiatric patients under maintenance electroconvulsive therapy: a one-year longitudinal study. J Neuropsychiatry Clin Neurosci 2004;16:465-471.

65 Kellner CH, Knapp RG, Petrides G, Rummans TA, Husain MM, Rasmussen K, Mueller M, Bernstein HJ, O'Connor K, Smith G, Biggs M, Bailine SH, Malur C, Yim E, McClintock S, Sampson S, Fink M: Continuation electroconvulsive therapy vs pharmacotherapy for relapse prevention in major depression: a multisite study from the Consortium for Research in Electroconvulsive therapy (CORE). Arch Gen Psychiatry 2006; 63:1337-1344.

-66 Navarro V, Gasto C, Torres X, Masana G, Penades R, Guarch J, Vazquez M, Serra M, Pujol N, Pintor L, Catalan R: Continuation/ maintenance treatment with nortriptyline versus combined nortriptyline and ECT in late-life psychotic depression: A two-year randomized study. Am J Geriatr Psychiatry 2008;16:498-505. 
-67 Smith GE, Rasmussen KG, Cullum CM, Felmlee-Devine MD, Petrides G, Rummans TA, Husain MM, Mueller M, Bernstein HJ, Knapp RG, O'Connor MK, Fink M, Sampson $\mathrm{S}$, Bailine SH, Kellner $\mathrm{CH}$ : A randomized controlled trial comparing the memory effects of continuation electroconvulsive therapy versus continuation pharmacotherapy: results from the Consortium for Research in ECT (CORE) study. J Clin Psychiatry 2010; 71:185-193.

68 Lisanby SH, Sampson S, Husain MM, Petrides G, Knapp RG, McCall V, Young RC, Prudic J, Kellner CH: Toward individualized post-electroconvulsive therapy care: piloting the symptom-titrated, algorithm-based longitudinal ECT (STABLE) intervention. J ECT 2008;24:179-182.
69 Barnes RC, Hussein A, Anderson DN, Powell D: Maintenance electroconvulsive therapy and cognitive function. Br J Psychiatry 1997; 170:285-287.

70 Beale M, Kellner C: Electroconvulsive therapy and drug interactions. Psychiatric Clin North Am: Annual Drug Ther 1996;3:119133.

71 UK ECT Review Group: Efficacy and safety of electroconvulsive therapy in depressive disorders: a systematic review and metaanalysis. Lancet 2003;361:799-808.

72 Pettinati HM, Stephens SM, Willis KM, Robin SE: Evidence for less improvement in depression in patients taking benzodiazepines during unilateral ECT. Am J Psychiatry 1990;147:1029-1035.
73 Bailine SH, Safferman A, Vital-Herne J, Bernstein S: Flumazenil reversal of benzodiazepine-induced sedation for a patient with severe pre-ECT anxiety. Convulsive Therapy 1994;10:65-68.

74 Kellner C, Burns C, Bernstein H, Monroe R: Electrode placement in maintenance ECT. Convuls Ther 1991;7:61-62.

75 Aziz M, Mehringer AM, Mozurkewich E, Razik GN: Cost-utility of 2 maintenance treatments for older adults with depression who responded to a course of electroconvulsive therapy: results from a decision analytic model. Can J Psychiatry2005;50:389397.

76 Dew R, McCall WV: Efficiency of outpatient ECT. J ECT 2004;20:24-25. 